\title{
Perfil socio-ocupacional de la población asistida en el equipo de gestión social territorial oeste - Mar del Plata
}

Socio-occupational profile in the population supported by the social management team at the Mar del Plata West district

Paola Yanina Cabero

Licenciada en Servicio Social

(Universidad Nacional de Mar del Plata)

Especialista en Violencia Familiar

(Universidad Nacional de Mar del Plata)

Correo: paola_yc@yahoo.com.ar,pycabero@gmail.com 


\title{
Resumen
}

En un escenario de crisis económica nacional, que sitúa a la ciudad de Mar del Plata entre las más afectadas desde el punto de vista socio-ocupacional, ubicándola, en al menos la última década, en los primeros lugares de desocupación a nivel nacional, y con índices de pobreza que alcanzan el 24,8\% de las personas, e indigencia del 6,5\% de personas (INDEC, 2018); nos proponemos en este trabajo compartir algunos resultados surgidos de la sistematización de parte de los datos recabados a lo largo de un año en la estadística de atención diaria en el Equipo de Gestión Social Territorial Oeste, para conocer el perfil sociolaboral de la población asistida y constatar si existe una brecha con otros estudios locales y nacionales. El estudio se realiza con perspectiva de género, haciendo hincapié en las desigualdades de género encontradas y ensayando algunas explicaciones que analizan cómo operan las representaciones sociales, los prejuicios y estereotipos en cada contexto histórico social. La Organización de las Naciones Unidas (1995) estableció que la incorporación de la perspectiva de género en los estudios y políticas redundaría en más oportunidades sociales y económicas, así como menos violencia de género.

\section{Palabras clave}

Perfil socio-ocupacional, Género, Políticas sociales.

\begin{abstract}
In this context of national economic crisis, that places the city of Mar del Plata between the more affected from a social - occupational point of view, at least in the last decade, reaching the first places of unemployment in national standards, poverty indicators that achieve $24,8 \%$ of the local people, and destitution of $6,5 \%$ of the people (according to INDEC measures in 2018), we propose in this work to share some of the emerging results based on the systematization of data gathered throughout one year of daily attention in the Western Territory of Social Management Team, to know the social-labour profile of the assisted population and determine if there is a gap with other national and local studies. The study was carried out from a gender perspective, emphasizing the inequality of gender findings and rehearsing some explanations that analyse how the social representation apparatus operates, prejudices and stereotypes in every social-historical context. The United Nations Organization (1995) established that the incorporation of gender perspectives in studies and policies would lead to more social and economic opportunities, as well as less gender violence.
\end{abstract}

Keywords

Social-professional profile, Gender, Social policy. 


\section{Introducción}

En este trabajo nos proponemos analizar cuali-cuantitativamente los datos que se producen cotidianamente en el Equipo de Gestión Social Territorial $^{1}$ Oeste (de ahora en más EGSTO), con el objetivo de obtener una aproximación del perfil sociodemográfico de las personas que concurrieron a solicitar orientación y/o asistencia al mismo, en el periodo comprendido entre el mes de septiembre 2018 y agosto de $2019^{2}$. Además, constituye la profundización de algunos aspectos abordados en la tesina de Posgrado de Especialización en Violencia Familiar ${ }^{3}$ presentada en 2018.

Entendemos que resulta imposible plantear estrategias de intervención desligadas del contexto socioeconómico nacional y local, y en particular, de la población con la que trabajamos, ya que de lo contrario corremos el riesgo de caer en abordajes superficiales, vaciados de mediaciones teóricas y que evidentemente no resolverán la problemática. Pero, además, contar con estos datos concretos, brinda la oportunidad de interpelar a las instituciones del Estados a las que pertenecemos, poniendo en agenda social, o al menos intentándolo, aspectos surgidos en nuestras prácticas cotidianas.

Como profesionales insertes laboralmente en el Estado, adquiere particular significado el carácter de las políticas públicas que en cierta medida les dan marco a las intervenciones, sin embargo, muchas veces éstas son

1 Los Equipos de Gestión Social Territorial dependen del Departamento de Gestión Social Territorial, de la Dirección de Promoción Social y Comunitaria, de la Secretaría de Desarrollo Social (MGP). Los mismos constituyen centros de atención primaria, no especializados, con un área de influencia geográficamente definida y con un carácter descentralizado. Por su concepción, son centros desde los cuales se vehiculiza la oferta pública de las políticas sociales municipales (Cabero, 2018). Es Misión de los EGST (Depto de Gestión Social Territorial, 2013) "La prevención de aquellas situaciones donde se vulneren derechos básicos y la promoción de capacidades, valores y potencialidades comunitarias y la asistencia individual y familiar." Y una de sus funciones "Realizar un diagnóstico actualizado de la zona de actuación de cada equipo profesional a través de una investigación permanente que permita definir los problemas sociales relevantes, las características generales de la población, los recursos comunitarios y todas aquellas variables que consideren pertinentes, permitiendo re-visionar la tarea en función de los nuevos escenarios territoriales".

2 En el marco del Seminario de Posgrado “Trabajo, género y políticas públicas. Una mirada interseccional en el marco de la crisis de la sociedad salarial" UNMDP. Facultad de Humanidades. Secretaria de Posgrado. 2019

3 Título: Violencia familiar: Propuestas para su visibilización. Un estudio desde el Equipo de Gestión Social Territorial Oeste de la ciudad de Mar del Plata. 2015/2016. 
antagónicas con el posicionamiento teórico, ético y político del profesional, tema que merece un mayor desarrollo, aunque excede el objetivo de este trabajo, configurando tensiones en el ejercicio profesional (Mallardi, Redondi y Moledda, 2018).

Por lo tanto, con este estudio pretendemos aportar un análisis crítico y con perspectiva de género de las variables seleccionadas (género, edad, nivel de instrucción, situación ocupacional) con la aspiración de contribuir a repensar las políticas públicas sociales municipales, donde predominan políticas homogeneizantes y de carácter focalizado, planificadas en función de carencias y ejecutadas en función de prestaciones (Programa Alimentario, Acción Social Directa, Mejoramiento Habitacional, entre otros) cuyos requisitos de acceso implica demostrar estado de necesidad de las personas y familias, negando su carácter de portadoras de derechos.

Estadísticas elaboradas desde el Depto. de Gestión Social Territorial en el primer semestre 2019, muestran que aproximadamente el 61\% de la población que concurre a los EGST lo hace en función de acceder al Programa de Complemento Alimentario Familiar ${ }^{4}$ o al Programa de Acción Social Directa $^{5}$, porcentajes similares a los que se observaron en el estudio anterior realizado desde este EGST (Cabero, 2018) donde el 74,7\% concurrió por "Problemas relacionados con la falta de alimentos adecuados". Considerando esos datos y basados en el conocimiento empírico, podemos inferir que la población del estudio, se encuentra encuadrada en situaciones de pobreza o indigencia según sus ingresos, y con altos índices de inactividad, desocupación y/o inestabilidad y precariedad laboral.

4 Programa Municipal "orientado a grupos familiares bajo la línea de indigencia, en situación de vulnerabilidad alimentaria”. El requisito incluye que el grupo familiar perciba ingresos por debajo del SMVM, que rondaba entre los $\$ 9000$ y $\$ 1100$ en el periodo de estudio. Se implementa mediante la entrega de una tarjeta precargada para compra de alimentos por un monto de $\$ 300$ en periodo de estudio.

5 Programa de Asistencia directa destinado a familias bajo línea de pobreza e indigencia tendiente a contribuir a la mejora de su calidad de vida con la gestión de prestaciones sociales y recursos (colchones, frazadas, zapatillas, entre otros). 


\section{Presentación y Análisis de datos}

La muestra analizada en el presente trabajo fue recolectada desde el EGST Oeste. El mismo cuenta con un área programática delimitada geográficamente al sur por la Av. Juan B. Justo, al norte por la Av. Libertad, al este por Av. Champagnat y al oeste por la calle Salvador Vivas, que corresponde con el fin del ejido urbano. A este rectángulo se suma el barrio Hipódromo [Imagen 1]

Esta zona presenta urbanísticamente características muy heterogéneas, constituyendo una de las zonas periféricas donde se concentra la población más pobre, y donde la infraestructura y provisión de servicios es más deficiente. Además, presenta un mayor crecimiento vegetativo, un índice de dependencia -relación entre personas laboralmente activas e inactivassuperior al del centro/microcentro/norte, e inferiores niveles de educación formal (Bucci, 2012).

La atención se realiza desde dos sedes municipales, Centro de Desarrollo Infantil (de ahora en más CDI) Jorge Newbery y La Herradura. Dentro de los límites de esta área quedan incluidos once barrios completos y parte de otros tres.

La fuente de datos utilizada en el estudio, es la planilla estandarizada de registro estadístico de atención diaria, que releva el motivo de la demanda, género y edad. A esta planilla base y general, en todos los EGST del Departamento de Gestión Social Territorial se anexó un registro de las variables "nivel educativo" y "situación laboral" durante el periodo de tiempo consignado (septiembre 2018 a agosto 2019).

\section{Análisis de datos}

En el periodo de referencia se tomaron un total de 1637 entrevistas $^{6}$, 1008 en la sede del CDI Newbery y 629 en la sede del barrio La Herradura.

6 Una persona puede haber concurrido en más de una oportunidad al servicio social en el periodo de tiempo consignado. Cada concurrencia se enumera de forma independiente. Por lo tanto, número de entrevistas, no equivale a número de personas. 
En primera instancia, podemos realizar dos primeras apreciaciones. Por un lado, se observa un progresivo crecimiento de la demanda en el transcurso de los meses de estudio [Gráfico 2]-; y si bien el presente trabajo no profundizará el análisis de forma específica, se realizarán algunas valoraciones de forma indirecta al analizar el perfil sociolaboral de la población atendida.

Por otro lado, se observa el predominio de concurrencia de población femenina, y en este aspecto sí nos detendremos. Se realizaron un total de 1286 entrevistas a mujeres, constituyendo el 78,6\% sobre el total de la muestra; 345 entrevistas a varones, que representa el 21\% y 6 entrevistas a mujeres $\operatorname{trans}^{7}(0,36 \%)$. Más específicamente, la población trans la constituyeron dos mujeres trans, cuyo perfil sociodemográfico se distinguirá en los análisis de las variables. Porcentajes similares encontramos en la estadística realizadas desde el Depto. de Gestión Territorial en el primer semestre 2019 que incluye todos los EGST de la ciudad. En la misma, se observa que se atendió un $72,4 \%$ de mujeres, $26,9 \%$ varones y $0,7 \% \mathrm{~S} / \mathrm{D}$.

Se devela un claro sesgo de género en la población que concurre a los EGST en general, y en Oeste en particular, cristalizándose cuestiones vinculadas a la división sexual del trabajo doméstico y la función atribuida a las mujeres (Cutuli, 2012). Además, se debe considerar que históricamente las políticas sociales de asistencia estuvieron dirigidas a la población excluida de la seguridad social, asociada al mundo del trabajo, población conformada principalmente por mujeres, niñes y ancianes; encontrándose de esta manera representades en el espacio público y, sin embargo, feminizadas (Cutuli, 2019).

Aun así, se observa una retracción de esta tendencia si consideramos el trabajo realizado por Cabero (2018) sobre una muestra tomada entre abril y septiembre de 2015 en el mismo EGST, donde el porcentaje de mujeres

7 Término paraguas que es utilizado con frecuencia para describir y agrupar múltiples identidades de género de las personas que tienen por común denominador que su sexo asignado al nacer no se alinea con su identidad reconocida. Por ende, no se incluye como criterio la presencia de transformaciones corporales, intervenciones quirúrgicas o tratamientos médicos (quirúrgicos y/u hormonales). El término mujeres trans se refiere a personas cuyo sexo asignado al nacer fue masculino mientras que su identidad de género es femenina. (Procuración Penitenciaria de la Nación. (2020). Mujeres trans privadas de libertad: la invisibilidad tras los muros. Recuperado de https://www.ppn.gov.ar) 
atendidas fue del 88,5\%. No obstante, dado que en este EGST se viene observando como constante la concurrencia de varones "solos", sin familia a cargo, se sostiene la hipótesis que este cambio no se debería a una progresiva equiparación en la división sexual del trabajo doméstico, sino a que, debido al deterioro de sus condiciones socio laborales (tema analizado más adelante) se han visto en la necesidad de demandar asistencia, aun cuando la prestación ofrecida (complemento alimentario) no se corresponde con la problemática planteada (desocupación, subocupación, ingresos insuficientes).

La distribución por edad/género de la muestra indica que tanto en mujeres como en varones el intervalo de 50-59 años es el que predomina con un 24,6\% y 24,5\% respectivamente; además de incluir a una de las mujeres trans. Mientras que el segundo intervalo que incluye la mayor cantidad de mujeres, 30-39 años con un 21,5\%, incluye la segunda mujer trans.

En el caso de las mujeres se observa que la distribución etaria es bastante equitativa en todos los intervalos hasta los 60 años, con un 17,5\% entre 18 y 29 años, 21,2\% de 40 a 49 años, cayendo al 11,2\% para mayores de 60 años. Se estima que esto deriva del acceso al beneficio previsional al que suscriben por la moratoria popularmente conocida como "jubilación de amas de casa" (Ley Nacional 26970), considerando los datos relativos a la registración laboral señalados en el análisis del punto siguiente.

En cambio, para los varones se observa un predominio de los últimos intervalos de edad, presentando un 15,6\% entre 18 y 29 años, $11 \%$ entre 30 y 39 años, 19,4\% de 40 a 49 años, 23,5\% de 60 a 69 años y 3,5\% de 70 años o más. Esta tendencia se asocia a la trayectoria laboral que, aun siendo inestable, se vuelve más excluyente a mayor edad, no pudiendo acceder al beneficio jubilatorio hasta los 65 años de edad, ya sea a través de moratorias o de la Pensión Universal Adulto Mayor (PUAM). Además, la mayoría de las políticas sociales vigentes, dado su carácter de focalización, apuntan en general a población que tiene bajo su responsabilidad niñes menores de 18 años de edad o con problemáticas específicas, quedando por fuera de los criterios de elegibilidad de las mismas. Del 3,9\% (mujeres) y 2,9\% (varones) no se cuenta con el dato. 
Se destaca que ambas mujeres trans se encuentran en rango de edad que supera el promedio de vida de dicha población, que ronda los 36 años de edad, 35 años en América Latina, 32 años en la Argentina (Procuración Penitenciaria de la Nación, 2020).

Respecto al nivel de instrucción [Gráfico 4] se tomaron como variables de clasificación: analfabetx, primaria incompleta (PI), primaria completa (PC), primaria en curso (Pec), secundaria incompleta (SI), secundaria completa (SC), secundaria en curso (Sec), terciario-universitario incompleto (Ter-Un I), terciario-universitario completo (Ter-Un C).

La mayoría de las personas entrevistadas tienen como máximo nivel educativo alcanzado la PC, siendo el 30,6\% en las mujeres y el 35,3\% en los varones. Este rango incluye también a una de las mujeres trans.

En segundo lugar, destaca el número de personas que no ha concluido la instrucción primaria (PI), incluyendo el 18,8\% de las mujeres y 23,1\% de los varones.

Respecto a la instrucción media, se observa que el 16,6\% de las mujeres y el 13\% de varones ha completado este nivel (SC), y no lo ha finalizado (SI) el 13,4\% de las mujeres y el 7\% de los varones. Este intervalo de nivel de instrucción incluye a la segunda mujer trans del estudio.

En relación a la población trans, si bien la muestra es pequeña, no se aleja de los resultados obtenidos en el estudio realizado a nivel nacional que concluye que en el nivel de SC y SI se centraliza la mayor cantidad de la población, sólo el 20\% terminó el nivel secundario (Procuración Penitenciaria de la Nación, 2020).

Otro aspecto que merece ser destacado es el nivel de analfabetismo encontrado, 1,6\% de la población femenina y el 2,9\% de la masculina. Según datos del Instituto Nacional de Estadística y Censos (de ahora en más INDEC) la tasa de analfabetismo total de Argentina (2010) es del 1,9\%, siendo del 1,9\% en mujeres y 2\% en varones (INDEC, 2020). Es decir, la mayoría de la población que comprende la muestra, cuenta con bajo nivel de instrucción, y si bien las líneas analíticas no son concluyentes al respecto, puede afirmarse que es uno de los indicadores a tomar en cuenta al momento de analizar la inserción laboral (aspecto analizado en el punto siguiente). 
Para el análisis de la situación ocupacional se pensó en principio en las categorías de Actividad-PEA/Inactividad-PNEA utilizados por el INDEC en la Encuesta Permanente de Hogares $\left(\mathrm{EPH}^{8}\right)$ a pesar de considerar que las mismas no incorporan la perspectiva de género y contribuyen a que se mantenga invisibilizada la importancia del trabajo doméstico en los aportes macroeconómicos (Actis Di Pasquale y Lanari, 2003; Cutuli, 2012; D’alessandro, 2016).

Corresponde realizar una observación respecto a la variable incorporada como "Hacemos Futuro"-HF/Salario Social Complementario ${ }^{10}$-SSC" ya que, por un error metodológico, en la estadística fue registrado solo el nombre del programa, sin informar su contraprestación y/o la situación ocupacional. Esto dificulta distinguir si esta persona se encuentra dentro de la PEA (desocupadxs, subocupadxs) o si se encuentra en PNEA (estudiantes). Para subsanar parcialmente esta dificultad, se realizará el análisis de PEA y PNEA incluyendo y excluyendo esta población de ambas categorías.

De las mujeres entrevistadas, el 48,7\% pertenecen a la PNEA $^{11}$, el 47,9\% a la PEA -incluyendo HFySSC-, mientras que, si se excluye la variable HFySSC de la PEA, el porcentaje varía a 51\% PNEA y 45,4\% PEA. Del $3,4 \%$ no se cuenta con el dato. [Gráfico 5]

Dentro de la PEA-Mujeres [Gráfico 6] destaca la variable Desocupada con el 44,9\%, incluyendo HFySSC, valor que asciende al 47,3\% si se excluyen las mujeres que perciben HFySSC.

8 Población Económicamente Activa (PEA) o Fuerza de Trabajo: Ocupadas, Subempleadas, Desocupadas. Población No Económicamente Activa (PNEA): comprende a todas las personas, sin consideración de edad, que no son "económicamente activas", en razón de: a) asistencia a institutos de educación; b) dedicación a trabajos en el hogar; c) jubilación o vejez; d) u otras razones como enfermedad o incapacidad, que pueden especificarse" (OIT 1988 en Neffa et al, 2014).

9 "HACEMOS FUTURO" surge de la unificación de "ARGENTINA TRABAJA" y "ELLAS HACEN". La reformulación de los programas busca que todos los participantes cumplan con tres contraprestaciones: actualización de datos, terminalidad educativa y formación integral. Hacemos Futuro fomenta el desarrollo de las personas para que tengan más oportunidades de insertarse en el mundo del trabajo. El programa les brinda herramientas para que los participantes puedan capacitarse y mejorar sus condiciones de empleabilidad.

10 El Salario Social Complementario es un plan social destinada a brindar asistencia y apoyo a trabajadores de la "economía popular".

11 Para la clasificación de la categoría PEA, dado que la mayoría de los entrevistadxs referían más de una actividad laboral-changas varias-, se tomó para el análisis la principal mencionada. 
Dentro del grupo Mujeres Ocupadas encontramos una multiplicidad de actividades [Gráfico 6] entre las que se destaca el porcentaje de Empleadas domésticas con el 49,8\% (con HF-SSC) o 54,9\% (sin HF-SSC), de estas solo el 2,4\% refiere encontrarse registrada. Datos muy alejados de lo que se observa a nivel nacional, que desde 2005 muestra un progresivo incremento en la registración para las trabajadoras en casas particulares con la implementación de la deducción de las cargas sociales de las trabajadoras del impuesto a las ganancias (Ley 26063) y posteriormente, en 2013, con el ingreso en vigencia del Régimen Especial de Contrato para el Personal de Casas Particulares (Ley 2684), pasando entre 2005 y 2006 de 3,8 a 9,4\% de registración, llegando al 23\% en el cuarto trimestre de 2014 (Pérez, Cutuli y Garazi, 2018).

Con un porcentaje mucho menor, encontramos la variable Cuidadora (ancianes y/o niñes) con el 8,3\% (con HF-SSC) o 9,1\% (sin HF-SSC), reproduciendo de esta manera en el mercado su rol doméstico. Pero, si consideramos que en la normativa vigente (Ley 26844) las cuidadoras se categorizan dentro del "personal de casas particulares", concluimos que al menos el $58 \%$ de estas mujeres ocupadas se desempeñan en este sector, cuyo nivel de registración general es inferior a la media (23\% aproximadamente) (Actis Di Pasquale y Lanari, 2003; Pérez, Cutuli y Garazi, 2018).

En tercer lugar, el 7,7\% se desempeña como vendedora ambulante -tomado sobre totales que incluyen HF-SSC. El resto de la muestra se dispersa en ocupaciones como asistente de geriátrico, costurera, peluquera, mucama, camarera, feriantes, empleada de comercio, artesana, cuidacoches, empleada en la industria del pescado, empleada en empresa de limpieza, empleada en quintas, auxiliar de escuela y "cirujeo" (recolección de la basura de elementos reciclables y/o re vendibles: cartón, vidrio, plástico, metales, entre otros).

La mayoría de estas mujeres podría comprenderse en la denominada subocupación horaria (Neffa, 2014). Si bien esta información no se relevó en la estadística, y por lo tanto no se puede llegar a un dato concluyente, podemos inferir alguna relación con la función de crianza y las dificultades que se les genera para afrontar el cuidado de lxs niñxs o personas a cargo (Aspiazu, Cutuli y Luena, 2015). En esta línea, Lupica $(2011,2013,2014)$ afirma en tra- 
bajos elaborados para el Observatorio de la Maternidad de Argentina que se percibe en los últimos años una mayor participación de las mujeres-madres en el mercado laboral, pero, aún así, existen desventajas a nivel laboral entre las mujeres con y sin hijos. En la misma línea, otros estudios afirman que la participación laboral de las mismas disminuye en la medida que aumenta el número de hijos (Contartese y Maceira, 2005; Novick, Rojo y Castillo, 2008; citados en Aspiazu, 2014).

Otra cuestión que no se puede pasar por alto se refiere al nivel de precariedad laboral presentada por la mayoría de la población entrevistada. En la muestra analizada, sólo diez (10) mujeres, es decir 0,6\%, refieren encontrarse laboralmente registradas, mientras que ningún varón mencionó esta condición. Nuevamente los datos muestran mayor precariedad que el promedio registrado en la ciudad, que en el segundo trimestre de 2019 indicaba que el porcentaje de asalariados no registrados era de 33,6\% (Informe Sociolaboral del Partido de Gral. Pueyrredón No 26, 2019). Para Actis Di Pasquale y Lanari (2015), la registración y la estabilidad laboral son indicadores de la calidad del empleo, dado que implican el grado de protección y la garantía de derechos ligados al sistema de seguridad social.

Las ramas de actividad donde encontramos registración fueron: empleada doméstica (4), asistente de geriátrico (1), empleada en empresa de limpieza (1), subsidio industria del pescado (1), mucama/hotelería (temporaria en mes de febrero) (1), auxiliar de escuela (suplencia) (2).

Por su parte, el 66,1\% de los varones se encuentra dentro de la PEA, y el 31,6\% dentro de la PNEA. No siendo significativo el cambio con o sin HF-SSC, $65,8 \%$ y $31,8 \%$ respectivamente. Del 2,3\% no se cuenta con el dato [Gráfico 7].

En la PEA varones [Gráfico 8], también se destaca la variable desocupado, con el 44,3\%. Al analizar el grupo de Varones Ocupados encontramos que el $26,7 \%$ se desempeña en actividades relacionadas con la construcción (albañilería, pintura), seguida con el 17,3\% cirujeo. Con porcentajes similares se ubican quienes refieren cortar pasto $(16 \%)$, cuidar coches $(14,7 \%)$ y tareas de panadería (10,7\%). También encontramos en menor medida otras formas de cuentapropismo en el marco de la economía informal (vendedo- 
res ambulantes, mecánicos), tratándose siempre de trabajos signados por la inseguridad y la inestabilidad.

$\mathrm{Al}$ analizar la PNEA Mujeres [Gráfico 9] se destaca con el 63,6\% la variable Ama de Casa -ADC-, completando la PNEA mujeres, un 24,8\% de pensionadas (no se distinguieron pensiones no contributivas por discapacidad y Madre de 7 hijos), un 9,7\% de jubiladas (incluye jubilaciones contributivas y PUAM) y un $1,7 \%$ de estudiantes.

Como la situación ya considerada en la subocupación, el análisis del indicador Ama de Casa requeriría ampliar conocimiento de otras variables, entre otras, la composición del grupo familiar (niñes, parejas, personas con discapacidad), para examinarla de forma interrelacionada con la cuestión referida a políticas públicas de cuidado, o su ausencia que constituye también una política. Igualmente, podemos arriesgar algunas conjeturas en esa línea, al precisar que en la actualidad el Estado municipal cuenta, para una población de 42.241 niños entre 0 y 4 años (INDEC, 2010), sólo con seis “casas del niño", ninguna en la zona de referencia, cuya cobertura total no supera a los setecientos niños. Solo tres de estas reciben niñes desde los 45 días a los 2 años, y resulta casi imposible conseguir cupo. En las últimas décadas no se ha creado ningún servicio nuevo:

(...) el área geográfica de mayor fragmentación, densidad poblacional y riesgo: la zona oeste en toda su traza, no cuenta con ningún servicio de cuidado ya que, los servicios existentes fueron inauguradas hace más de 3 décadas y en razón del crecimiento de la ciudad, han quedado en barrios que hoy exhiben mayores niveles de integración y accesibilidad urbana que los del sector oeste (Bucci y Bucci, 2017).

En tanto, según datos del Ministerio de Educación "la oferta de cuidado infantil para menores de dos años en su totalidad es privada y se concentra en 19 instituciones" (Cutuli, 2019:235). Además, la autora destaca que la modalidad de media jornada educativa de la escolaridad obligatoria no se compatibiliza con los tiempos de trabajo requeridos a los adultos, madres en particular, sobre quienes recae la responsabilidad de cuidado en general. 
Al mismo tiempo, también se podría inferir la existencia de violencia de género en el ámbito familiar, en cualquiera de sus tipos, donde el varón no permite la salida al mundo laboral de esa mujer como el resultado de una modalidad de relación basado en las asimetrías de poder. Esta relación es altamente factible si consideramos los datos del Registro Único de Casos de Violencia contra las Mujeres (de ahora en más RUCVM) (INDEC, 2019) que señalan un promedio doscientos sesenta y tres casos de violencia de género denunciados por día en la Argentina ante diversos organismos públicos, correspondiéndose el 82,1\% con un vínculo de pareja o expareja.

Otro aspecto observado al momento de tomar entrevistas a las mujeres que en ese momento no trabajan fuera del hogar, es que se definen como amas de casas, y al indagar, categorizarían como desocupadas, ya que se encuentran buscando trabajo remunerado, o como subocupadas, ya que realizan "changas" desde la casa (costura, elaboración de alimentos, eventualmente concurren a una feria). De este modo, la estadística invisibilizaba no sólo el trabajo femenino sino la desocupación femenina, en tanto que cuando no trabajaban para el mercado, eran amas de casa "por defecto" (Cutuli, 2012).

Además, hemos distinguido a una mujer que no puede trabajar por sus condiciones de salud, y por ello la definimos en la variable denominada "inactiva". Esta, a su vez, no puede acceder a una pensión no contributiva, por ser inmigrante que no cumple con requisito de años de residencia para acceder al beneficio. Queda en manifiesto la desprotección para aquella población que no cumple con las condiciones propuestas por Castel (1997) para definir la relación salarial y su inclusión en las políticas de protección social.

Por su parte, en la PNEA Varones sobresale la variable pensionado que representa el $77 \%$, seguido por jubilados con el 16,5\% y estudiantes $0,9 \%$. El alto porcentaje de esta población que percibe una pensión no contributiva, cuyo ingreso representa el $70 \%$ de la jubilación mínima, siendo aproximadamente de $\$ 9000$ en junio 2019, con ingresos que apenas equiparan el monto de índice de pobreza para una persona (en el primer semestre 2019 la Canasta Básica Alimentaria para un adulto ronda los $\$ 4000$ según datos del INDEC, y que no siempre cuentan con otras fuentes de ingreso estable, 
da cuenta de la alta vulnerabilidad social, económica y sanitaria de esta población).

También hemos distinguido la variable denominada "inactivo" que, como en la situación de las mujeres, no pueden trabajar por problemas de salud, aunque por diferentes circunstancias aún no cuentan con la pensión. Insistimos en este punto, la diferencia notable es que, mientras que la mujer que "no trabaja para el mercado" por el motivo que sea, se suele percibir (por ella misma o por quienes) toman la encuesta, como "ama de casa" (Cutuli, 2012). El varón en situación similar se define (por él mismo o por quienes toman la encuesta) como "desocupado", aunque técnicamente para serlo debería haber buscado activamente trabajo, constituyendo un hecho con claro sesgo de género y que tiene consecuencias estadísticas, ya que en el primer caso se toma dentro de la PNEA y en el otro en la PEA.

Por último, analizamos la situación ocupacional de las mujeres trans de la muestra, que aun siendo tan pequeña, requiere un análisis específicos por las características particulares de vulneraciones que muestra este colectivo en el acceso a la educación, el empleo, la salud y la vivienda, factores que las conlleva, a menudo, a trabajar en economías informales altamente criminalizadas, como el mercado de drogas, el trabajo sexual o el sexo por supervivencia (Procuración Penitenciaria de la Nación, 2020).

La primera de ellas percibe una pensión no contributiva por discapacidad, por patologías físicas y psicológicas tras una historia de vida de discriminación y precariedad laboral. La segunda también presenta evidentes secuelas físicas y psicológicas (ataques de pánico que impiden salir de la casa), pero aún no ha podido gestionar la pensión. En este caso, para la subsistencia, además de recibir ayuda de parte de su familia, elabora alimentos en su domicilio, comercializándolos una familiar.

La experiencia de ambas no escapa a la realidad que vive la mayoría de la población trans en la Argentina y la región (Cutuli, 2015). Esta realidad evidencia la necesidad de políticas públicas integrales o de articulación efectiva entre ellas, para el abordaje de las múltiples problemáticas expresadas por esta población, que habilite la restitución de los derechos que le han sido negados a lo largo de sus vidas. 


\section{Discusión}

La ciudad de Mar del Plata es la cabecera del Partido de General Pueyrredón. Según datos del Censo 2010 tiene una población estable de 618.989 habitantes compuesto por 323.695 mujeres y 295.294 varones, superando la población de nueve provincias argentinas. El $95 \%$ se concentra en la ciudad de Mar del Plata, es decir, se trata de un partido eminentemente de población urbana, que representa el 3,9\% de los habitantes del total de la provincia de Buenos Aires.

Sus características sociodemográficas, de acuerdo con la estructura de edades por grandes grupos (0-14 años, 15 a 64 años y 65 y más años), presenta una población envejecida, dada la creciente y elevada participación de la población adulta mayor de 65 años y más $(14,2 \%)$, superando los guarismos a nivel provincial y nacional de 10,69 \% y 10,23 \% respectivamente (INDEC, 2010).

Con relación al empleo y al perfil productivo, Actis Di Pasquale (2018) señala que la ciudad presenta sus propias características, siendo las principales la estacionalidad y las elevadas tasas de desocupación y subocupación.

La estructura productiva del partido de Gral. Pueyrredón muestra un fuerte predominio de las actividades del sector terciario, destacándose por su peso relativo las actividades comerciales con un $25 \%$, las actividades inmobiliarias, empresariales y de alquiler con un $22 \%$, y el transporte con un $15 \%$, todas ellas influenciadas a lo largo de todo el año, en buena medida, por la actividad turística, aún cuando esta presenta variaciones estacionales y no ha tenido en los últimos años su mejor desempeño (Graña et al, 2019).

La pesca constituye la principal actividad económica dentro del sector primario local, siendo otro de los sectores productivos tradicionales que se destacan en la ciudad, encontrándose, además, ligada a la historia y los orígenes de la ciudad. Es uno de los motores industriales, aunque se halla en declinación, entre otras, como consecuencia del agotamiento del recurso pesquero (Rearte, 2002). Concomitantemente, ha padecido en las últimas décadas la precarización laboral, entre otras formas, a través de las pseudo cooperativas (Cutuli, 2018). 
El cordón frutihortícola de Mar del Plata, además de autoabastecerla, provee de sus productos a otras regiones, constituyendo uno de los principales abastecedores del país en frutas, hortalizas, cereales, oleaginosas, así como en la cría de ganado bovino y ovino. No obstante, en este sector productivo se destacan altos niveles de explotación laboral (cuasi-esclavitud), ocupando mano de obra migrante, principalmente boliviana, en muchos casos, en situación de ilegalidad.

En el sector secundario, la industria manufacturera está representada prioritariamente por tres ramas de actividad, alimentación, textil y metalmecánica, las que componen aproximadamente el 80\% del valor agregado total de la industria local. En el parque industrial "General Savio" se radican más de 60 fábricas, dedicándose alrededor del $40 \%$ a la producción de alimentos (Graña et al, 2019).

A nivel nacional, y vinculado al empleo y al poder adquisitivo de acuerdo a los ingresos, se destaca para los 31 aglomerados medidos por INDEC (2019) en el primer semestre de 2019 un porcentaje de hogares por debajo de la línea de pobreza del 25,4\%, comprendiendo al 35,4\% de las personas. Dentro de este conjunto se distingue un 5,5\% de hogares indigentes, que incluyen el 7,7\% de las personas. Los indicadores mencionados registran una suba de la pobreza y la indigencia con respecto al segundo semestre de 2018 y también respecto de los consignados en el primer semestre de 2018.

Para la ciudad de Mar del Plata, en el segundo semestre del 2018, encontramos valores que ubican al $18,6 \%$ de hogares y $24,8 \%$ de personas por debajo de la línea de pobreza, y un 5,2\% de hogares y $6,5 \%$ de personas por debajo de la línea de indigencia. Mientras que en el primer semestre de 2019 el 20,5\% de hogares y 29,5\% de las personas están por debajo de la línea de pobreza, y el 3,5\% de hogares y el 6,3\% de personas se encuentran bajo la línea de indigencia.

De acuerdo a los datos aportados por el $\operatorname{GrET}^{12}(2018,2019)$ para el periodo de estudio, en la ciudad observamos un deterioro significativo del 
mercado de trabajo, con incremento de la desocupación y subocupación, con altos porcentajes de trabajo no registrado, presentando valores similares a los observados en 2004 (salida de la crisis socioeconómica del 2001).

En tal sentido, para el $4^{\circ}$ trimestre de 2018 (inicio del periodo de estudio) se observa una caída del trabajo registrado del 1,2\% interanual a nivel nacional. La tasa de desocupación presentó significativos aumentos en el plano nacional, donde pasa del 7,2\% al 9,1\%, mientras que a nivel local el incremento es más pronunciado, pasando del 9,3\% al 12,8\%, ubicando a nuestra ciudad, junto con el aglomerado Gran Rosario, con la mayor tasa de desocupación del país. La tendencia se conserva en los informes siguientes manteniéndose en el primer trimestre 2019 en 10,1\%, aun considerando la temporada estival, segundo trimestre $13,4 \%$, mientras que en el tercer trimestre se produce un descenso al 10,2\%, aunque se mantiene en dos dígitos.

Otro aspecto destacado en estos informes, señala que a pesar que Mar del Plata se caracterizaba por su estacionalidad y su mayor índice de asalariados en los primeros trimestres, esta tendencia estaría desapareciendo, ubicando en 2019 a "Mar del Plata en el segundo lugar de los aglomerados con menos trabajo en relación de dependencia detrás de Salta $(65,3 \%)$ y seguido por Gran Rosario (67,4\%)" (Informe Sociolaboral No 25. 2019:10). Mientras que, para el segundo trimestre de 2019, afirma que se presenta el peor escenario laboral desde fines de 2016:

La caída del empleo y el aumento de la desocupación se dieron en todos los grupos de edad comprendidos entre los 14 y 64 años. Y dado el aumento de la tasa de actividad que también fue generalizado y llegó a niveles récord. Recordemos que las mujeres habían incrementado su participación hasta el tercer trimestre de 2018 inclusive, con la finalidad de complementar los ingresos del hogar, ocupándose en trabajos precarios o del sector informal, o bien, como cuentapropistas. El último dato disponible da cuenta de que este efecto es masivo y no distingue ni por sexo ni por grupos de edad (Informe Sociolaboral No 26. 2019:10).

En este contexto, resulta fundamental conocer cómo se articulan las políticas de protección social y las de protección al desempleo, y cómo, en un país territorialmente tan diverso y extenso, se ejecutan las mismas, coordi- 
nando los diferentes programas implementados desde los niveles nacionales, provinciales y municipales, evitando la superposición, en pos de la eficiencia en el uso de los recursos (Bertranou y Paz, 2007). Sobre todo, considerando que históricamente los sistemas de protección, en Latinoamérica en general y Argentina en particular, se ligaron a la situación ocupacional de las personas, siendo de carácter residual las políticas de asistencia. Pero particularmente, en gobiernos neoliberales, como el reinante en el periodo de estudio, donde predominan las prestaciones circunscriptas a grupos de individuos, aislados, empobrecidos y que se encuentran fuera del mercado formal de trabajo (Piñero y Fernández, 2014).

En Mar del Plata, las políticas de asistencia implementadas a través de los EGST se encuentran focalizadas y ejecutadas con lógicas de selectividad hacia las poblaciones definidas como pobres. Esto es así a pesar que la misión de dichos equipos supone la prevención de aquellas situaciones donde se vulneren derechos básicos y la promoción de capacidades, valores y potencialidades comunitarias y la asistencia individual y familiar (Depto. de Gestión Social Territorial, 2013), quedando de esta manera, las intervenciones promocionales ligadas a los esfuerzos individuales de cada profesional.

Por su parte, las políticas de mercado de trabajo y de protección al desempleo (Bertranou y Paz, 2007) a nivel local se piensan de manera divorciada de las de asistencia, y se han caracterizado por estar dirigidas a la capacitación y entrenamiento para mejorar la empleabilidad de la población, con algunos programas específicos y focalizados en poblaciones acotadas (Jóvenes con Más y Mejor Trabajo y/o Promover La Igualdad de Empleo) articulados con el gobierno nacional. Además, dispone de una "bolsa de trabajo", posibilitando la carga de C.V., que buscaría el enlace de la oferta y demanda laboral, y de un área destinada al acompañamiento de micro emprendedores, que también ve supeditada su labor a la implementación de programas provinciales y nacionales.

\section{Conclusiones}

El trabajo tuvo como objetivo general realizar una aproximación al perfil sociodemográfico de la población que concurrió al EGST Oeste entre los 
meses de septiembre 2018 a agosto 2019, partiendo de la conjetura de que la población atendida concurre a solicitar asistencia encontrándose, en general, debajo de la línea de pobreza.

Del análisis de los datos obtenidos en las entrevistas realizadas se desprende, en primera instancia, un incremento progresivo de la cantidad de personas que concurrieron al EGST Oeste a solicitar asistencia u orientación. Esta circunstancia si bien no puede ser corroborada con los datos recolectados, la atribuimos al empeoramiento de la situación socio ocupacional de la población, detallada a lo largo del trabajo y ampliada en apartado de discusión.

En segundo término, se destacó el absoluto predominio de concurrencia de población femenina, que relacionamos con la división sexual del trabajo de cuidado, que incluye la asistencia a un espacio público para el acceso a las políticas públicas de asistencia social. Al mismo tiempo, el incremento del número de varones asistidos, comparado con trabajos previos, lo atribuimos, en base a la observación empírica, a que la mayoritaria de estos varones viven solos, sin personas a cargo ni que se ocupen de realizar estas gestiones, inferencia que podría ser el objetivo de siguientes estudios.

En referencia a la edad de la población, tanto en mujeres (incluyendo una mujer trans) como en varones, la mayoría se encuentra dentro de la franja de 50 a 59 años de edad. En el resto de las franjas etarias, en el caso de las mujeres la distribución es equitativa en todas edades estudiadas. Mientras que en varones se da mayor concurrencia en la franja de 50-59, seguida por 60-69 y 40-49. No podemos más que interrelacionar este dato con su precaria situación ocupacional, y aunque los datos del INDEC señalan que es la población joven, 20-29 años, donde se concentra la mayor tasa de desocupación, en nuestra experiencia se trata de población mayor, probablemente porque entre los jóvenes el desaliento a la hora de buscar trabajo sea menor, o porque no ven en la asistencia social una alternativa para solucionar este problema. Una tercera opción sería, volviendo a la división sexual del trabajo, que son jóvenes con pareja y son las mujeres quienes concurren a solicitar ayuda.

Resulta preocupante lo referente al bajo nivel de instrucción encontrado, ya que la mayoría de la población de la muestra se concentra en el nivel de 
instrucción primario, completo e incompleto, con un 49,4\% de las mujeres y $58,4 \%$ de los varones. A su vez, se considera alarmante el nivel de analfabetismo encontrado, especialmente en la población masculina, con un 2,9\%, porcentaje que supera la tasa de analfabetismo total de Argentina que es del 1,9\%, y 2\% en varones (INDEC, 2010). En una ciudad que presenta una amplia oferta de establecimientos educativos en todos sus niveles (excepto maternal como ya se señaló), y que ostenta, según el Censo de Población 2010, altos índices de instrucción terciaria y universitaria, el 7,9\% y 12,1\% respectivamente cursa o curso niveles superiores, valores sólo superados en la provincia de Buenos Aires por Bahía Blanca (13,7\%) y La Plata (22,9\%) (INDEC, 2010).

En referencia a la situación laboral, en la PEA predomina la desocupación con el 44,9\% en las mujeres y 44,3\% en los varones; y en el caso de los ocupados, encontramos trabajos realizados en contextos de precariedad, de duración intermitente, de baja calificación y remuneración, y de forma no registrada )sólo encontramos en mujeres un 0,6\% de registración laboral).

En tanto en la PNEA, destacamos el alto porcentaje de la variable "ama de casa" en las mujeres, y especulamos que uno de los factores que podrían incidir en este porcentaje se relaciona con la ausencia de políticas públicas de cuidado que tiendan a la desfeminización y desfamiliarización de la tarea. También encontramos altos índices de inactividad por situaciones relacionadas a la salud (pensionados), con ingresos que no cubren las necesidades básicas.

En conclusión, este trabajo nos presenta una foto que muestra una población con ingresos por debajo de la línea de pobreza, con bajo nivel de instrucción formal, altos índices de inactividad, desocupación y precariedad laboral. A partir de la misma, nos planteamos algunos interrogantes con la intención de pensar qué políticas sociales son necesarias y factibles para enfrentar la complejidad de situaciones que se presentan. Claramente, son necesarias políticas que alienten la terminalidad educativa y la formación laboral, apuntando al mejoramiento del capital social y cultural. Pero resulta pertinente la pregunta: ¿es factible que esta cantidad de personas logren in- 
sertarse, aún con capacitaciones que les brinden las herramientas necesarias, en el mercado de trabajo de forma regular?

En un sistema económico-social que plantea el trabajo asalariado -para el mercado- como medio privilegiado de acceso a la protección social, cabe preguntarse qué tipo de políticas sociales se requieren para afrontar el contexto planteado en el presente trabajo. En esta línea Romina Cutuli señala en referencia a las políticas públicas "que tímidamente esbozaron una escisión entre trabajo y derechos e ingresos, siguen provocando fuertes disensos de corte moralizante $(. .$.$) ni siquiera cuando se reconoce abiertamente que es$ creciente la población que nunca alcanzará la quimera del "trabajo decente"” (2019:29).

No tenemos las respuestas, sí la intención de poner en tensión algunas prácticas instaladas por el Estado (Nacional, Provincial, Municipal) desde sus políticas sociales. También tenemos el compromiso ético político de hacer visible el escenario territorial descripto en nuestro estudio, sin perder de vista que las problemáticas planteadas en relación a la subsistencia, vivienda, educación y salud, en su sentido más amplio, deben ser abordadas desde la perspectiva de la asistencia social como derecho (Campana, 2017) y no como "beneficiarios" de políticas sociales-asistenciales focalizadas, dirigidas a poblaciones pobres.

\section{Referencias bibliográficas}

Actis Di Pasquale, Eugenio y Lanari, Marcos Esteban (2003). Asimetrías entre géneros en el mercado laboral marplatense. VII Jornadas Nacionales de Historia de las Mujeres y II Congreso Iberoamericano de Estudios de Género. Salta, Argentina.

Actis Di Pasquale, Eugenio y Lanari, Marcos Esteban (2018). Las tasas básicas del mercado de trabajo. Conceptos y formas de cálculo. Material de cátedra de Introducción a la Economía. Facultad de Ciencias Económicas y Sociales, Universidad Nacional de Mar del Plata. Recuperado de: http://nulan.mdp.edu.ar/2874/1/actis-2018.pdf

Actis Di Pasquale, Eugenio y Lanari, Marcos Esteban (2019). Informe Sociolaboral del Partido de General Pueyrredon, (24) Facultad de Ciencias Económicas y Sociales, Universidad Nacional de Mar del Plata. Recuperado de: http://nulan.mdp.edu.ar/3103/1/InfoSociolabMar2019.pdf

Actis Di Pasquale, Eugenio y Lanari, Marcos Esteban (2019). Informe Sociolaboral del Partido de General Pueyrredon, (25) Facultad de Ciencias Económicas y Sociales, Universidad Nacional de Mar del Plata. Recuperado de: http://nulan.mdp.edu.ar/3188/1/InfoSociolabLun2019.pdf 
Actis Di Pasquale, Eugenio y Lanari, Marcos Esteban (2019). Informe Sociolaboral del Partido de General Pueyrredon, (26) Facultad de Ciencias Económicas y Sociales, Universidad Nacional de Mar del Plata. Recuperado de: http://nulan.mdp.edu.ar/3256/1/InfoSociolabSep2019.pdf

Actis Di Pasquale, Eugenio y Lanari, Marcos Esteban (2019). Informe Sociolaboral del Partido de General Pueyrredon, (27) Facultad de Ciencias Económicas y Sociales, Universidad Nacional de Mar del Plata. Recuperado de: http://nulan.mdp.edu.ar/3286/1/InfoSociolabDic2019.pdf

Actis Di Pasquale, Eugenio; Lanari, María Estela y Hasanbegovic, Claudia (2015). "El mercado laboral escenario de desigualdades persistentes. Las brechas abiertas de Argentina". En M. E. Lanari, y C. Hasanbegovic (Ed.), Mujeres de Latinoamérica. El presente en veintidós letras. Mar del Plata, Argentina, EUDEM

Aspiazu, Eliana; Cutuli, Romina y Luena, María Teresa (2015). La igualdad en la conciliación entre trabajo y cuidado: una revisión de las regulaciones vigentes. VI Encuentro Regional de Estudios del Trabajo. Tandil, Argentina.

Aspiazu, Eliana (2014). Conciliación entre trabajo y responsabilidades familiares: una revisión teórica con enfoque de género. Investigium IRE: Ciencias Sociales y Humanas, (1), 177-194.

Bertranou, Fabio y Paz, Jorge (2007). Políticas y programas de protección al desempleo en la Argentina. Argentina, Oficina Internacional del Trabajo.

Bucci, Laura (2012). Cohesión social y territorio: algunas reflexiones a partir de la experiencia del proyecto EU-LA-WIN- programa URB-AL III. Mar del Plata, Argentina, Ediciones Suárez.

Bucci Laura y Bucci Ines (2017). Los alcances y limitaciones de la protección social para mujeres que atraviesan situaciones de violencia. Un análisis de caso en la ciudad de Mar del Plata. III Jornadas Internacionales. Sociedad, Estado y Universidad. UNMDP.

Cabero, Paola (2018). Violencia familiar: Propuestas para su visibilización. Un estudio desde el Equipo de Gestión Social Territorial Oeste de la ciudad de Mar del Plata. 2015/2016 (Trabajo Final Integrador) Especialización en Violencia Familiar, Universidad Nacional de Mar del Plata, Argentina.

Campana, Melisa (2017). Asistencia Social y restauración neoliberal. Cátedra Paralela, (14), 13-24.: https://rephip.unr.edu.ar/bitstream/handle/2133/14237/Campana.pdf?sequence $=3$ \&is Allowed $=y$

Castel, Robert (1997). "La Sociedad Salarial”. En R. Castel (Ed.), La metamorfosis de la cuestión social. Una crónica del salariado (pp. 271-323). Barcelona, España, Paidós.

Cutuli, María Soledad (2015). Entre el escándalo y el trabajo digno. Etnografía de la trama social del activismo travesti en Buenos Aires. (Tesis doctoral). Recuperado de: http://repositorio.filo.uba.ar/bitstream/handle/filodigital/4610/uba_ffyl_t_2015_909398.pdf?sequen$\underline{\mathrm{ce}=1 \text { \&is Allowed }=\mathrm{y}}$

Cutuli, Romina Denisse (2012). Flexibilidad empresarial y organización del trabajo doméstico: el trabajo invisible de las hijas de las fileteras en Mar del Plata. La ventana. Revista de estudios de género, 4(36), 178-223.

Cutuli, Romina Denisse y Aspiazu, Eliana (2015). "Las políticas de cuidado infantil en Argentina. Aportes para su clasificación y evaluación". En M. E. Lanari y C. Hasanbegovic 
(Eds.), Mujeres de Latinoamérica. El presente en veintidós letras (pp. 339-371). Mar del Plata, Argentina, EUDEM.

Cutuli, Romina Denisse (2019). Del trabajo a la casa... Trabajo, mujeres y precarización laboral en la industria pesquera marplatense 1990-2010. Mar del Plata, Argentina, Eudem.

Cutuli, Romina Denisse (2012). Medir es conocer: Economía feminista y cuantificación del trabajo. Observatorio Laboral Revista Venezolana, 5(9), 23-41.

D’alessandro, Mercedes (2016). Economía feminista: cómo construir una sociedad igualitaria (sin perder el glamour). Editorial Sudamericana.

EPH (2018). Mercado de trabajo. Tasas e indicadores socioeconómicos. Trabajo e ingresos, 3(1)1 https://www.indec.gob.ar/uploads/informesdeprensa/mercado trabajo eph 4trim18.pdf

EPH (2019). Incidencia de la pobreza y la indigencia en 31 aglomerados urbanos. Condiciones de vida, 3(13). INDEC. https://www.indec.gob.ar/uploads/informesdeprensa/eph pobreza_01_19422F5FC20A.pdf

EPH (2019). Incidencia de la pobreza y la indigencia en 31 aglomerados urbanos. Condiciones de vida, 4(4) INDEC. https://www.indec.gob.ar/uploads/informesdeprensa/eph pobreza_02_195EFE752E31.pdf

Graña, Fernando Manueal, Gonzalez Barros, Ariel (Eds.) (2019). Mapa productivo. Estudio global de las empresas industriales del Partido de General Pueyrredon. Universidad Nacional de Mar del Plata, Argentina. Recuperado de: http://nulan.mdp.edu.ar/3275/1/mapa-productivo-pgp-2019.pdf

Instituto Nacional de Estadísticas y Censos (INDEC) (2010). Censo Nacional de Población, Hogares y Viviendas. República Argentina. INDEC. Recuperado de: https://www.indec. gob.ar/ftp/cuadros/poblacion/censo2010 tomo1.pdf

Instituto Nacional de Estadísticas y Censos (INDEC)(2019). Registro único de casos de violencia contra las mujeres-RUCVM: resultados 2013-2018. Ciudad Autónoma de Buenos Aires, Argentina, INDEC. Recuperado de: https://www.indec.gob.ar/uploads/informesdeprensa/rucvm_03 19.pdf

Lupica, Carina (2012). Trabajadoras domésticas ¿Cómo cuidamos a quienes nos proporcionan cuidados? Fundación Observatorio de la Maternidad. Boletín de la Maternidad (16), 2-7.

Lupica, Carina y Cogliandro, Gisell (2011). Las desventajas por la maternidad en el mercado de trabajo: un desafío pendiente. Congreso Nacional de Estudios del Trabajo. Fundación Observatorio de la Maternidad, Ciudad de Buenos Aires, Argentina.

Lupica, Carina (2013). Madres y padres jóvenes en Argentina: Su participación desigual en los estudios, el mercado de trabajo y el cuidado de los hijos. Newsletter del Observatorio de la Maternidad, (71).

Lupica, Carina (2014). Niñez, juventud y maternidad en la Argentina: interrelación de derechos y oportunidades a través del sistema de cuidado infantil. Newletter del Observatorio de la Maternidad, (78), 1.

Mallardi Manuel, Redondi Valeria y Moledda Marcela (2018). El proceso dialógico en el ejercicio profesional. Seminario Semi-presencial de capacitación y actualización profesional. $3^{\circ}$ 
Encuentro Trabajo Social: implicancias de la dimensión investigativa en el proceso dialógico. ICEP. CTSyAS.

Neffa, Julio. (2010). "Aportes para comprender la lógica de generación del empleo precario”. En A. Del Bono y G. Quaranta (Eds.), Convivir con la incertidumbre: Aproximaciones a la flexibilización y precarización del trabajo en la Argentina. Buenos Aires, Argentina, CEIL-PIETTE.

ONU Mujer (2017). Hechos y cifras: Acabar con la violencia contra mujeres y niñas. Recuperado de: http://www.unwomen.org/es/what-we-do/ending-violence-against-women/ facts-and-figur

Pérez, Ines; Cutuli Romina. y Garazi, Débora (2018). Senderos que se bifurcan: servicio doméstico y derechos laborales en la Argentina del Siglo XX. Mar del Plata, Argentina, EUDEM.

Piñero Fernando y Fernández Emiliano (2014). La política social en la actualidad latinoamericana: ¿hacia una política asistencial "pos-neoliberal"? Un análisis de las Transferencias Monetarias Condicionadas. Cátedra Paralela, (11), 50-77. http://www.catedraparalela.com.ar

Procuración Penitenciaria de la Nación (2020). Mujeres trans privadas de libertad: la invisibilidad tras los muros. Recuperado de https://www.ppn.gov.ar

Pizarro, Roberto (2001). La vulnerabilidad social y sus desafíos: una mirada desde América Latina. Santiago de Chile, Chile, CEPAL.

Recibido: 26/01/2021

Aceptado: 31/03/2021 


\section{Anexo}

Imagen 1: Ciudad de Mar del Plata- Área Programática EGST Oeste

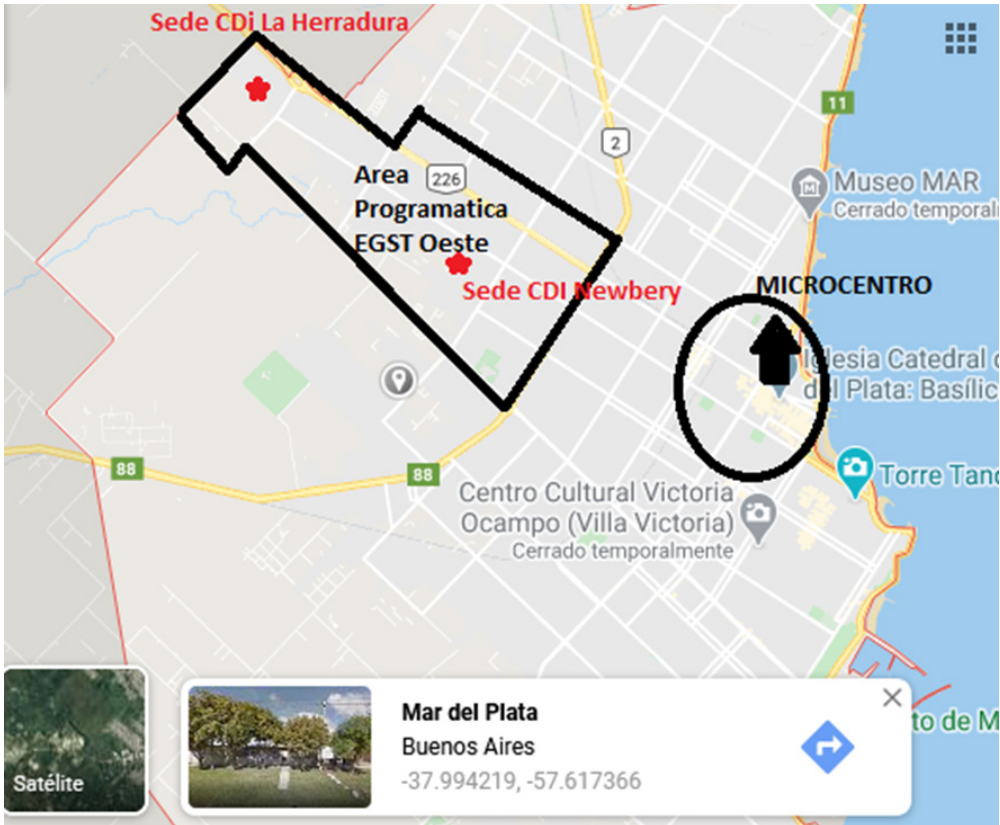

Gráfico 1- (En el mes de Diciembre 2018 se realizó una medida de fuerza que redujo significativamente el número de entrevistas realizadas.)

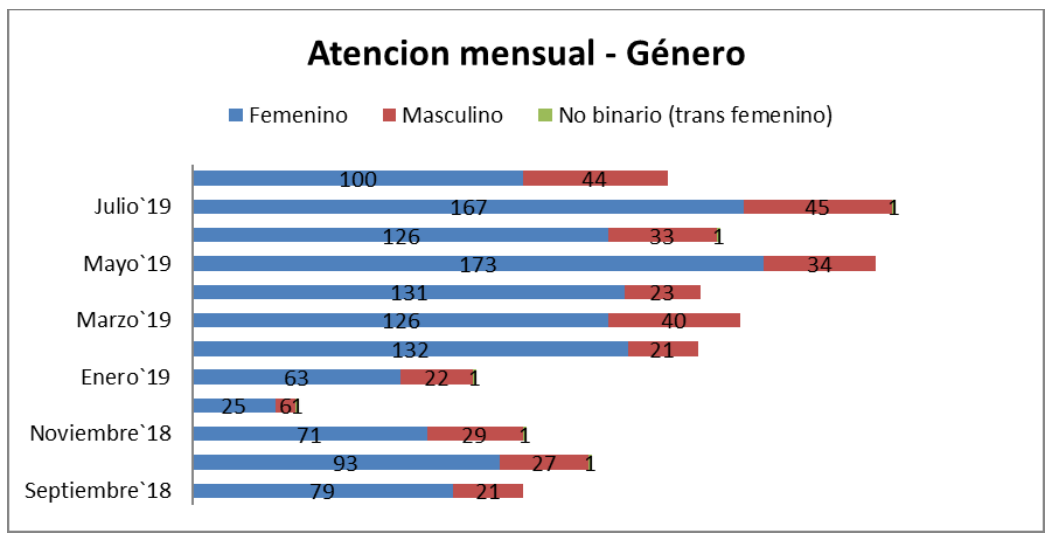


Gráfico 2

\section{Género}

Mujeres Varones Trans Femenino

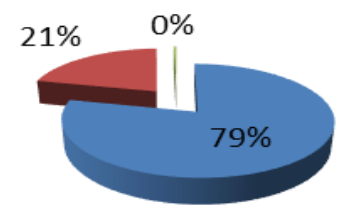

Gráfico 3

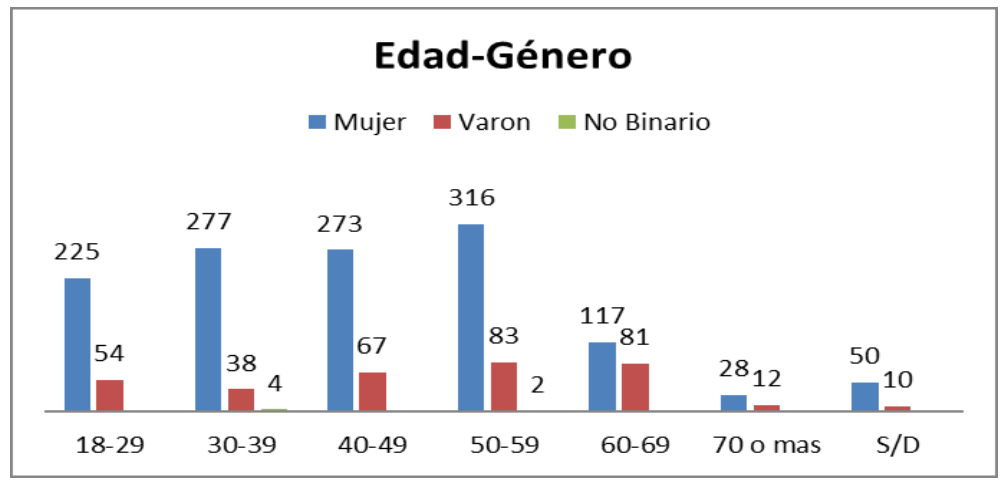

Gráfico 4

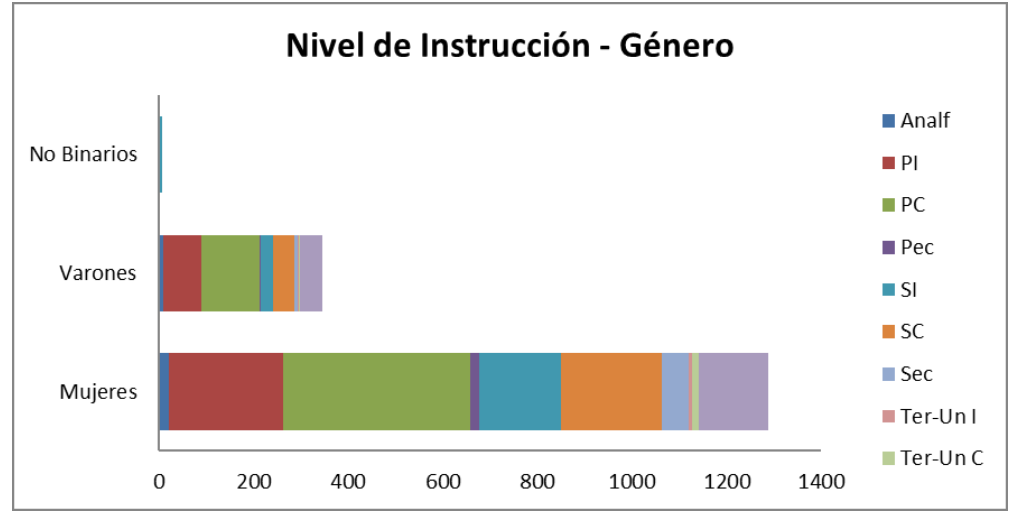


Gráfico 5

\section{Condición de Actividad- Mujeres}

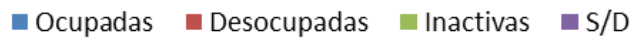

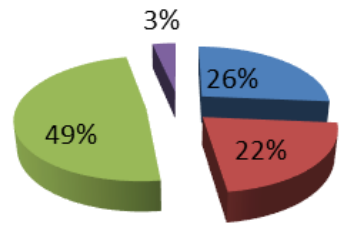

Gráfico 6

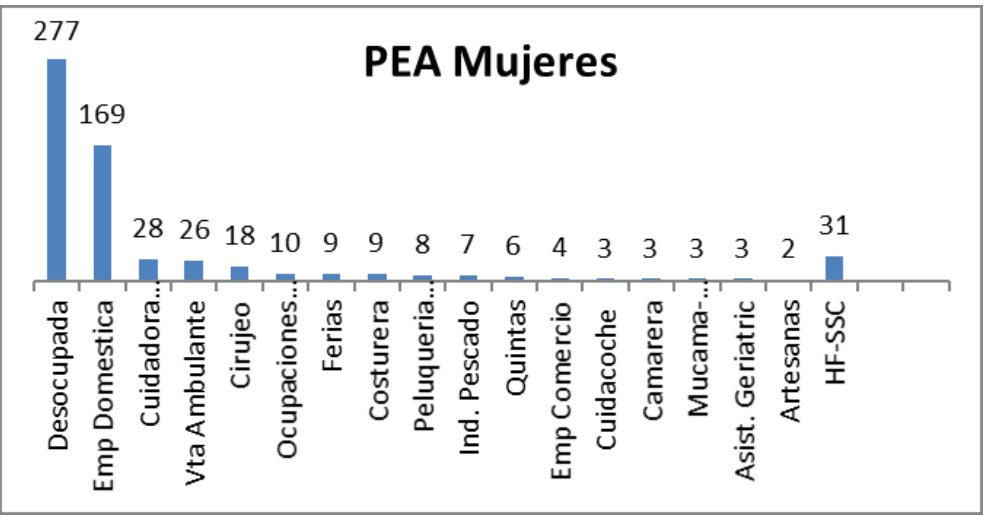

Gráfico 7

\section{Condición de Actividad- Varones}

- Ocupados $\square$ Desocupados $\square$ Inactivos $\square$ S/D

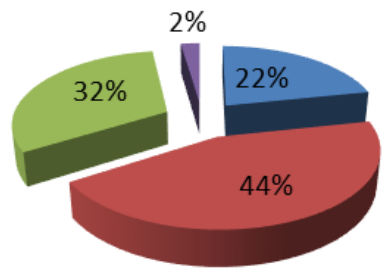


Gráfico 8

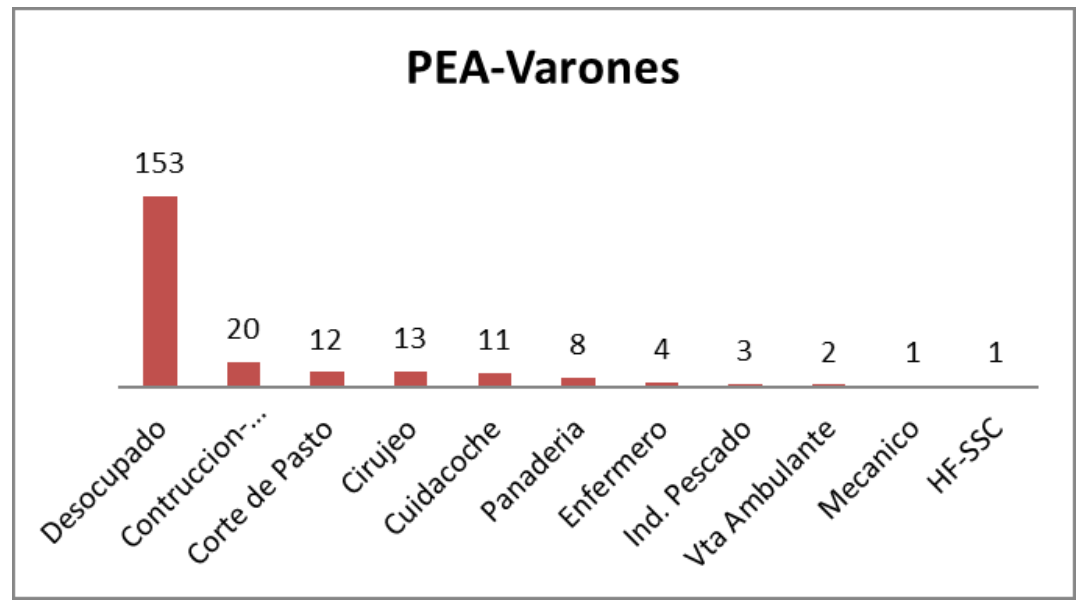

Gráfico 9

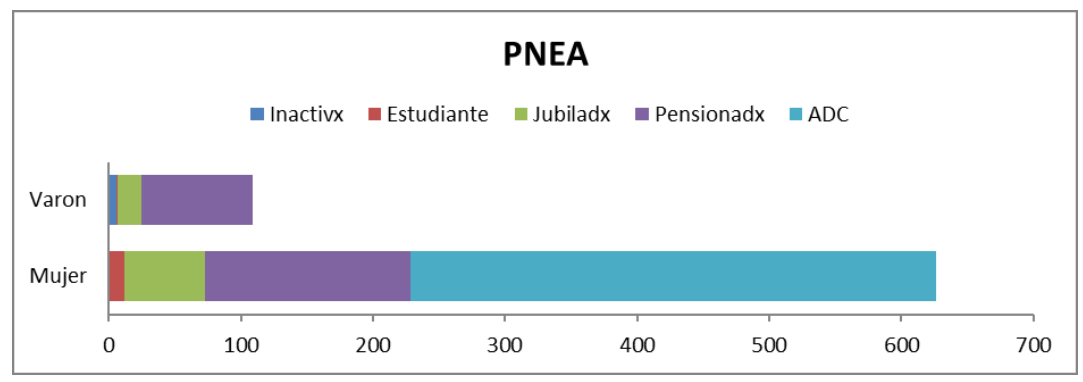

